\title{
Especialização em Saúde Pública: Os Alunos da Universidade de São Paulo no Período 1985-1994
}

\author{
Maria da Penha Costa Vasconcellos ${ }^{1}$ \\ Paulo Capel Narvai ${ }^{1}$
}

\begin{abstract}
Resumo: Este artigo aborda algumas características dos profissionais de saúde, alunos do Curso de Especialização em Saúde Pública mantido pela Universidade de São Paulo (USP). O período do estudo abrange os anos de 1985 a 1994. Foram analisadas as variáveis: ano de realização do curso, período de aulas, vínculo institucional, categoria profissional, sexo, idade, cidade de origem, macrorregião brasileira de origem e país de origem. Os dados foram colhidos junto à Seção de Alunos da Faculdade de Saúde Pública da USP, através de instrumento concebido para essa finalidade. Foi montado banco de dados e utilizado programa específico para os procedimentos estatísticos. Os resultados mostraram significativas alterações nas características dos alunos ao longo do período considerado, destacando-se o progressivo afastamento da categoria profissional de médicos e o crescente interesse dos assistentes sociais. A pesquisa evidenciou também que a USP, outrora formadora de especialistas em Saúde Pública vindos das mais diferentes macrorregiões brasileiras e mesmo do exterior, não tem conseguido manter tal abrangência.
\end{abstract}

Palavras-chave: Ensino de Saúde Pública; Especialização em Saúde Pública; Pós-Graduação lato sensu; Recursos Humanos em Saúde.

Summary: This article deals with some characteristics of the professionals who attend the Course of Specialization in Public Health maintained by the University of São Paulo (USP). The study comprehends the years 1985 to 1994 . The following variables were analyzed: year in which the course was carried out, period of classes, institutional link, professional category, gender, age, hometown, Brazilian macro-region and/or country of origin. The data were collected at the Students Section of the School of Public Health of USP, through an instrument conceived for this purpose. A database was created and a program specific for the statistical proceedings was used. The results pointed out significative changes in the students' characteristics during the period under analysis, notably the progressive decrease in the number of physicians and the growing interest of the social workers. The research also revealed that USP, formerly responsible for the formation of specialists in Public Health coming from many Brazilian macro-regions and even from abroad, has not been able to maintain this situation.

Keywords: Public Health Teaching; Specialization in Public Health; Lato sensu Post-Graduation; Human Resources in Health. São Paulo

1 Professores do Departamento de Prática de Saúde Pública da Faculdade de Saúde Pública, Universidade de 


\section{Introdução}

A Faculdade de Saúde Pública (FSP) da Universidade de São Paulo (USP) vem tendo, ao longo deste século, papel central no ensino da Saúde Pública no Brasil. Antes mesmo da criação da USP (1934), houvera sido criado, em 1925, o "Instituto de Higiene de São Paulo", sob a direção de Geraldo Horácio de Paula Souza que em 1922 havia assumido o cargo de professor titular do "Laboratório de Higiene", uma Cadeira criada em 1918 na Faculdade de Medicina, em decorrência de convênio firmado entre o governo do Estado de São Paulo e o International Health Board, da Fundação Rockefeller. Ao lado de Francisco Borges Vieira, com quem integrou a primeira turma de diplomados da School of Hygiene and Public Health da Universidade Johns Hopkins, dos Estados Unidos, Paula Souza instalou, em 1925, como anexo ao Instituto de Higiene, o primeiro Centro de Saúde do Brasil, que funcionou na rua Brigadeiro Tobias, $\mathrm{n}^{\mathrm{o}} 45$, constituindo um verdadeiro "laboratório" de ensino de Saúde Pública. Em 1931, o Instituto foi oficialmente denominado "Escola de Higiene e Saúde Pública", a qual se instalou, a partir de 1932, nas dependências onde hoje funciona a FSP-USP (Candeias, 1989; Krausz, 1977; Mascarenhas, 1951; Universidade de São Paulo, 1994; Vasconcellos, 1995). Em 1938, a Escola de Higiene e Saúde Pública foi incorporada à USP, tornando-se, segundo Kisil (1994), a primeira escola de Saúde Pública da América Latina a pertencer a uma Universidade; em 1945, teve sua denominação alterada para "Faculdade de Higiene e Saúde Pública" e, em 1969, para "Faculdade de Saúde Pública".

Cabe registrar que, no mesmo período em que ocorriam essas iniciativas em São Paulo, também no Rio de Janeiro, então Capital da República, era instituído, através do Decreto 16.782-A, de 13/01/1925, o "Curso de Higiene e Saúde Pública”, o primeiro destinado a formar médicos sanitaristas (Labra, 1985).
O primeiro curso do Instituto de Higiene de São Paulo teve início em 1925 e se destinava a professores primários. Foi o "Curso de Educadores Sanitários". Os médicos chegaram logo depois para o "Curso de Especialização em Higiene e Saúde Pública para Médicos". Após dois anos de estudos, a primeira turma de médicos sanitaristas diplomouse em 1929. Em 1941, após reconhecimento desse curso pelo Governo Federal, sua duração passou a seguir o padrão federal, reduzindo-se para um ano. Em 1949 foi instalado o "Curso de Especialização em Saúde Pública para Engenheiros"; em 1954, o "Curso de Especialização em Saúde Pública para Veterinários"; em 1958, o "Curso de Especialização em Saúde Pública para Cirurgiões-Dentistas"; e, em 1959, o "Curso de Especialização em Saúde Pública para Enfermeiros" (Candeias, 1984; Vasconcellos, 1995; Vasconcellos \& Narvai, 1994).

O curso de Pós-Graduação stricto sensu em Saúde Pública teve início na FSP-USP, em 1970, em decorrência da Reforma do Ensino Superior no país (Krausz, 1977; Universidade de São Paulo, 1994). A importância do papel que a FSP vem desempenhando no ensino nesse nível pode ser avaliada pelo expressivo número de candidatos às vagas oferecidas. Estudando a demanda para mestrado e doutorado em saúde coletiva, Tanaka (1996), observou que $35 \%$ dos candidatos pretenderam fazer mestrado ou doutorado na FSP-USP. Outra importante instituição nessa área, a Escola Nacional de Saúde Pública (ENSP), ficou com a preferência de $20 \%$ do total de candidatos. Em conjunto, FSP e ENSP atraíram $55 \%$ dos que aspiraram fazer seus estudos de Pós-Graduação em Saúde Coletiva.

A atuação da FSP-USP tem sido decisiva, em todos os sentidos, desde os primeiros cursos, na formação e desenvolvimento de recursos humanos nesse campo, atingindo a maior parte dos países da América Latina e os de língua portuguesa da África. Em 1982, os diferentes cursos específicos de especiali- 
zação em saúde pública segundo a graduação do aluno foram unificados em um "Curso de Especialização em Saúde Pública” (CESP). Desde então, sucessivas modificações foram introduzidas e o CESP foi adquirindo o formato atual (1996), cujas características principais são: multiprofissionalidade; duração de um ano; aulas diárias, das 8 às 12 horas; disciplinas gerais comuns, no primeiro semestre; disciplinas específicas organizadas em áreas temáticas/especialidades, no segundo semestre, com divisão dos alunos segundo essas áreas e reagrupamento dos alunos na parte final para a realização de trabalhos de campo multiprofissionais. O CESP objetiva à formação de sanitaristas capacitados para a gestão tanto de sistemas de saúde de qualquer complexidade, quanto das diferentes unidades ou áreas técnicas que os constituem. As mudanças que têm marcado a evolução do Sistema de Saúde brasileiro nas últimas décadas têm implicado mudanças no CESP, na tentativa de fazer com que os egressos estejam em sintonia com os serviços para os quais se dirigem; e de onde a maioria provêm (Vasconcellos \& Narvai, 1994).

A par das dificuldades inerentes à busca dessa "sintonia", as próprias transformações do Sistema de Saúde brasileiro têm influenciado, de modo significativo, o interesse de diferentes profissionais pela "especialização em Saúde Pública”. O foco desse artigo está nesses profissionais, com o objetivo de compreender algumas de suas características e, dessa forma, contribuir para que o CESP ou outros cursos similares possam estar adequados às necessidades da população e do Sistema de Saúde brasileiro.

\section{Material e Método}

O material compõe-se de um conjunto de fichas obtidas junto à Seção de Alunos da FSP-USP, preenchidas pelos candidatos ao CESP, por ocasião da inscrição para seleção.
As fichas se referem ao período 1985-1994. Dessas fichas, foram obtidos dados de cada aluno através de instrumento concebido exclusivamente para essa finalidade. Esses dados, devidamente codificados, foram inseridos num banco de dados, a partir do qual foram processados e tratados estatisticamente. As informações obtidas se referem a algumas características dos profissionais de saúde, alunos do CESP. Foram consideradas as seguintes variáveis: a) ano de realização do curso (1985 a 1994); b) periodo de aulas (integral ou apenas matutino); c) área temátical especialidade (enfermagem, veterinária, odontologia, laboratório em saúde pública, medicina, educação em saúde, engenharia em saúde pública, biologia, nutrição, engenharia ambiental, saúde mental, ciências sociais em saúde, advocacia em saúde, saúde maternoinfantil, epidemiologia, políticas, planejamento, organização e gerenciamento de serviços de saúde, vigilância sanitária, administração de serviços de saúde-básica, administração de serviços de saúde-avançada); d) vínculo institucional (administração direta - municipal, estadual ou federal; administração indireta - municipal, estadual ou federal; setor privado - lucrativo ou não lucrativo); e) categoria profissional; f) gênero; g) idade; h) cidade de origem; i) estado de origem; j) macrorregião brasileira de origem; k) país de origem.

\section{Resultados e Discussão}

Os resultados obtidos são apresentados nas tabelas numeradas de 1 a 7 e gráficos numerados de 1 a 3 .

Quanto à origem (Tabela 1), os alunos do CESP foram majoritariamente $(96,6 \%)$ provenientes do Brasil. Houve 2 europeus (1 francês e 1 belga $-0,2 \%$ ) e 15 africanos $(1,4 \%)$, no período. Os latino-americanos de língua espanhola corresponderam a 1,6\% dos alunos. 
Tabela 1

Distribuição dos Alunos do Curso de Especialização em Saúde Pública da FSP-USP, segundo o País de Origem, no Período 1985-94

\begin{tabular}{l|rr}
\hline \multicolumn{1}{c|}{ Pais de Origem } & \multicolumn{1}{c}{ № } & $\%$ \\
\hline Angola & 3 & 0,3 \\
Bélgica & 1 & 0,1 \\
Bolívia & 2 & 0,2 \\
Brasil & 977 & 96,6 \\
Costa Rica & 1 & 0,1 \\
Equador & 2 & 0,2 \\
França & 1 & 0,1 \\
Guatemala & 2 & 0,2 \\
Moçambique & 12 & 1,1 \\
Panamá & 2 & 0,2 \\
Paraguai & 3 & 0,3 \\
Peru & 3 & 0,3 \\
Uruguai & 1 & 0,1 \\
Sem informação & 2 & 0,2 \\
\hline Total & 1.012 & 100,0 \\
\hline
\end{tabular}

Tabela 2

Distribuição dos Alunos do Curso de Especialização em Saúde Pública da FSP-USP, segundo a Macrorregião de Origem, no Período 1985-94

\begin{tabular}{l|cc}
\hline Macrorregião de Origem & № & $\%$ \\
\hline Norte & 13 & 1,3 \\
Nordeste & 20 & 2,0 \\
Centro-Oeste & 3 & 0,3 \\
Sudeste & $937\left(^{*}\right)$ & 95,8 \\
Sul & 4 & 0,4 \\
Sem informação & 2 & 0,2 \\
\hline Total & $979\left(^{* *}\right)$ & 100,0 \\
\hline
\end{tabular}

* São Paulo = $928(94,7 \%)$.

** Apenas os brasileiros.

Acrescentando-se os 33 estrangeiros $=1.012$ alunos.

Observa-se na Tabela 2 que os brasileiros vieram predominantemente do Estado de São Paulo $(928=94,7 \%)$. A região sudeste representou $95,8 \%$ do total. Dentre as demais macrorregiões, destacou-se o nordeste com
20 alunos $(2,0 \%)$. O agrupamento "norte-nordeste" superou o agrupamento "sul-centrooeste": $3,3 \%$ e $0,7 \%$, respectivamente.

Os dados apresentados na Tabela 3 indicam que a mudança do período de realização de atividades do CESP (integral ou apenas matutino) não produziu modificações significativas quanto à atração de alunos provenientes de outras localidades que não São Paulo; fossem eles da região metropolitana de São Paulo ou de outras regiões. Por outro lado, verifica-se na Tabela 4 que as mudanças no período de realização do curso parecem ter estimulado a participação de alunos oriundos da "administração direta municipal", cuja participação percentual interna às categorias elevou-se de 22,4\% (integral) para 40,3\% (matutino). As demais categorias não se alteraram significativamente, à exceção da "administração direta estadual", que caiu de $48,5 \%$ para $35,3 \%$. Contudo, como o período do estudo coincide com uma notável diminuição do quadro de servidores da Secretaria de Estado de Saúde de São Paulo e uma expansão no número de profissionais de saúde vinculados aos municípios, é possível que este seja o fator relevante e não o período de realização das atividades do CESP.

Aproximadamente $10 \%$ dos alunos do CESP eram do sexo masculino, vinculados profissionalmente ao setor privado (Tabela 5). Quando o vínculo profissional era com o setor público essa percentagem aumentava sem, contudo, ultrapassar a um terço. De modo geral, o sexo feminino predominou em todas as categorias consideradas. A exceção ficou por conta dos estrangeiros, onde o sexo masculino alcançou a proporção de $60,6 \%$ do total de alunos desse grupo.

Observa-se na Tabela 6 que o maior número de alunos está registrado na categoria de médicos $(273=27,0 \%)$. Esses profissionais são seguidos pelos enfermeiros (127 $=12,6 \%)$, pelos cirurgiões-dentistas (103 = $10,3 \%)$, e pelos graduados em serviço social $(86=8,5 \%)$. Há de se considerar, todavia, 
Tabela 3

Distribuição dos Alunos do Curso de Especialização em Saúde Pública da FSP-USP, segundo as Doze Cidades de Origem com Maior Freqüência e o Período de Realização do Curso. São Paulo, 1985-94

\begin{tabular}{l|rr|r}
\hline \multirow{2}{*}{\multicolumn{1}{c|}{ Cidade de Origem }} & \multicolumn{2}{|c|}{ Período } & \multirow{2}{*}{ Total } \\
\cline { 2 - 3 } São Paulo & Integral & Matutino & 740 \\
Santo André & 438 & 302 & 12 \\
Guarulhos & 11 & 1 & 13 \\
Maputo & 1 & 12 & 10 \\
Santos & 6 & 4 & 10 \\
Marilia & 6 & 4 & 9 \\
São Bernardo do Campo & 7 & 2 & 8 \\
Araraquara & 6 & 2 & 7 \\
Osasco & 6 & 1 & 7 \\
Bauru & 6 & 1 & 5 \\
Campinas & 3 & 2 & 3 \\
São José do Rio Preto & 2 & 1 & 3 \\
\hline
\end{tabular}

Tabela 4

Distribuição dos Alunos do Curso de Especialização em Saúde Pública da FSP-USP, segundo Vínculo Profissional e Período. São Paulo 1985-94

\begin{tabular}{|c|c|c|c|c|c|c|}
\hline \multirow{3}{*}{ Vínculo Profissional } & \multicolumn{4}{|c|}{ Período } & \multirow{2}{*}{\multicolumn{2}{|c|}{ Total }} \\
\hline & \multicolumn{2}{|c|}{ Integral } & \multicolumn{2}{|c|}{ Matutino } & & \\
\hline & $\mathrm{N}^{\circ}$ & $\%$ & № & $\%$ & № & $\%$ \\
\hline Administração Direta Municipal & 146 & 22,4 & 145 & 40,3 & 291 & 28,7 \\
\hline Administração Direta Estadual & 316 & 48,5 & 127 & 35,3 & 443 & 43,8 \\
\hline Administração Direta Federal & 6 & 0,9 & 2 & 0,6 & 8 & 0,8 \\
\hline Administração Indireta Municipal & 1 & 0,2 & - & - & 1 & 0,2 \\
\hline Administração Indireta Estadual & 39 & 6,0 & 19 & 5,3 & 58 & 5,7 \\
\hline Administração Indireta Federal & 44 & 6,7 & 22 & 6,1 & 66 & 6,5 \\
\hline Setor Privado Não Lucrativo & 6 & 0,9 & 4 & 1,1 & 10 & 1,0 \\
\hline Setor Privado Lucrativo & 26 & 4,0 & 17 & 4,7 & 43 & 4,2 \\
\hline Estrangeiro & 25 & 3,8 & 8 & 2,2 & 33 & 3,3 \\
\hline Sem informação & 43 & 6,6 & 16 & 4,4 & 59 & 5,8 \\
\hline Total & 652 & 100,0 & 360 & 100,0 & 1.012 & 100,0 \\
\hline
\end{tabular}

que ao longo do período sob análise os médicos têm sua participação sensivelmente diminuída, enquanto aumenta a percentagem de graduados em serviço social (Gráfico 1). Também é expressivo o crescimento dos psicólogos, conforme demonstrado na Tabela 7.
No que diz respeito à idade dos alunos do CESP (Gráfico 3), a média foi de 33 anos, a moda 31 e a mediana 32. O desvio-padrão foi de 6 anos. Tais valores indicam que, de um modo geral, os profissionais procuraram se especializar cerca de uma década após 
Tabela 5

Distribuição dos Alunos do Curso de Especialização em Saúde Pública da FSP-USP, segundo Vínculo Profissional e Gênero, no período 1985-94

\begin{tabular}{|c|c|c|c|c|c|c|}
\hline \multirow{3}{*}{ Vínculo Profissional } & \multicolumn{4}{|c|}{ Gênero } & \multirow{2}{*}{\multicolumn{2}{|c|}{ Total }} \\
\hline & \multicolumn{2}{|c|}{ Feminino } & \multicolumn{2}{|c|}{ Masculino } & & \\
\hline & № & $\%$ & № & $\%$ & № & $\%$ \\
\hline Administração Direta Municipal & 241 & 82,8 & 50 & 17,2 & 291 & 100,0 \\
\hline Administração Direta Estadual & 341 & 77,0 & 102 & 23,0 & 443 & 100,0 \\
\hline Administração Direta Federal & 6 & 75,0 & 2 & 25,0 & 8 & 100,0 \\
\hline Administração Indireta Municipal & 1 & 100,0 & - & - & 1 & 100,0 \\
\hline Administração Indireta Estadual & 43 & 72,9 & 15 & 27,1 & 59 & 100,0 \\
\hline Administração Indireta Federal & 46 & 69,7 & 20 & 30,3 & 66 & 100,0 \\
\hline Setor Privado Não Lucrativo & 9 & 90,0 & 1 & 10,0 & 10 & 100,0 \\
\hline Setor Privado Lucrativo & 38 & 88,4 & 5 & 11,6 & 43 & 100,0 \\
\hline Estrangeiro & 13 & 39,4 & 20 & 60,6 & 33 & 100,0 \\
\hline Sem informação & 50 & 84,7 & 7 & 15,3 & 59 & 100,0 \\
\hline Total & 788 & 77,9 & 222 & 22,1 & 1.012 & 100,0 \\
\hline
\end{tabular}

Gráfico 1

Número de Médicos e Assistentes Sociais Alunos do Curso de Especialização em Saúde Pública da Universidade de São Paulo, no Período 1985-1994

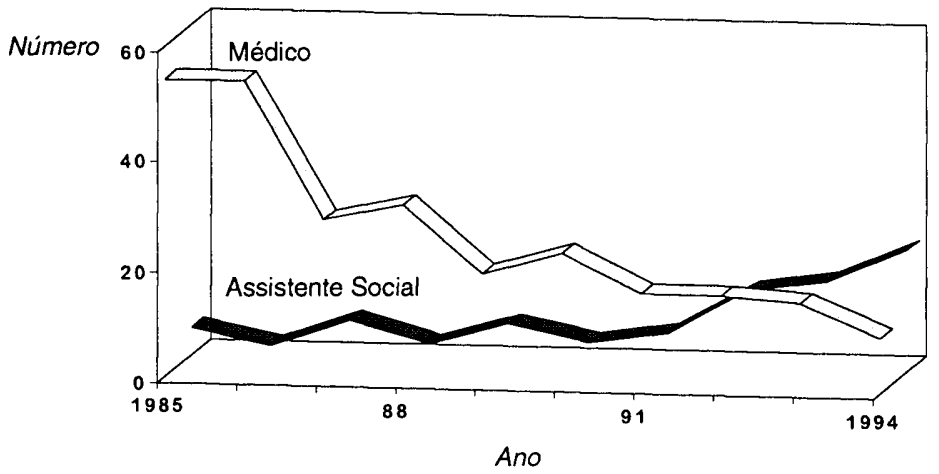

Gráfico 2

Número e Porcentagem de Alunos do Curso de Especialização em Saúde Pública da Universidade de Sāo Paulo Segundo Sexo, no Período 1985-1994

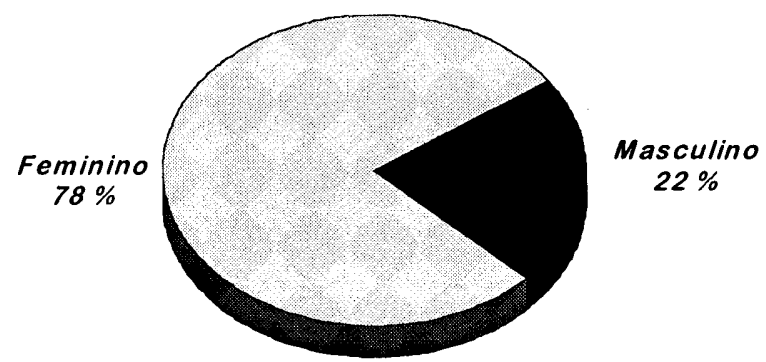


Tabela 6

Distribuição dos Alunos do Curso de Especialização em Saúde Pública da FSP-USP, segundo o Curso de Graduação, no Período 1985-94

\begin{tabular}{|c|c|c|}
\hline Curso de Graduação & No & $\%$ \\
\hline Administração de Empresas & 2 & 0,2 \\
\hline Arquitetura & 2 & 0,2 \\
\hline Biblioteconomia & 1 & 0,1 \\
\hline Biologia & 64 & 6,4 \\
\hline Biomédica & 6 & 0,5 \\
\hline Ciências Biológicas & 11 & 1,0 \\
\hline Ciências Contábeis & 1 & 0,1 \\
\hline Ciências Políticas & 1 & 0,1 \\
\hline Ciências Sociais & 23 & 2,4 \\
\hline Comunicação Social & 5 & 0,4 \\
\hline Desenho Industrial & 1 & 0,1 \\
\hline Direito & 14 & 1,4 \\
\hline Ecologia & 2 & 0,2 \\
\hline Economia & 2 & 0,2 \\
\hline Economia Doméstica & 1 & 0,1 \\
\hline Educação Social & 1 & 0,1 \\
\hline Enfermagem & 127 & 12,6 \\
\hline Engenharia & 3 & 0,3 \\
\hline Estatística & 2 & 0,2 \\
\hline Farmácia & 60 & 5,9 \\
\hline Filosofia & 1 & 0,1 \\
\hline Fisioterapia & 8 & 0,8 \\
\hline Fonoaudiologia & 8 & 0,8 \\
\hline Geografia & 4 & 0,4 \\
\hline História & 1 & 0,1 \\
\hline Jornalismo & 1 & 0,1 \\
\hline Letras & 3 & 0,3 \\
\hline Matemática & 4 & 0,4 \\
\hline Medicina & 273 & 27,0 \\
\hline Medicina Veterinária & 47 & 4,6 \\
\hline Nutrição & 23 & 2,3 \\
\hline Odontologia & 103 & 10,3 \\
\hline Ortóptica (Profissionalizante) & 1 & 0,1 \\
\hline Pedagogia & 35 & 3,4 \\
\hline Psicologia & 56 & 5,5 \\
\hline Publicidade & 1 & 0,1 \\
\hline Química & 3 & 0,3 \\
\hline Segundo Grau Profissionalizante & 1 & 0,1 \\
\hline Serviço Social & 86 & 8,5 \\
\hline Sociologia & 7 & 0,7 \\
\hline Técnico em Medicina Preventiva (Profissionalizante) & 2 & 0,2 \\
\hline Técnico em Saúde Pública (Profissionalizante) & 3 & 0,3 \\
\hline Técnico em Zootecnia (Profissionalizante) & 1 & 0,1 \\
\hline Terapia Ocupacional & 7 & 0,7 \\
\hline Sem informação & 4 & 0,3 \\
\hline Total & 1.012 & 100,0 \\
\hline
\end{tabular}


Tabela 7

Distribuição dos Alunos do Curso de Especialização em Saúde Pública da FSP-USP, segundo o Curso de Graduação, no Período 1985-94

\begin{tabular}{l|rrrrrrrrrrrrr}
\hline \multirow{2}{*}{ Curso de Graduação } & \multicolumn{7}{|c}{ Ano } & \multicolumn{10}{c}{ Total } \\
\cline { 2 - 12 } Medicina & 85 & 86 & 87 & 88 & 89 & 90 & 91 & 92 & 93 & 94 & NF & $\%$ \\
Enfermagem & 54 & 54 & 29 & 32 & 20 & 24 & 17 & 17 & 16 & 10 & 273 & 29,6 \\
Odontologia & 17 & 6 & 14 & 23 & 8 & 8 & 6 & 22 & 15 & 8 & 127 & 13,7 \\
Serviço Social & 11 & 12 & 10 & - & 13 & 11 & 6 & 11 & 19 & 10 & 103 & 11,2 \\
Biologia & 5 & 2 & 7 & 3 & 7 & 4 & 6 & 14 & 16 & 22 & 86 & 9,3 \\
Farmácia & 8 & 8 & 6 & 7 & 8 & - & 7 & 9 & 8 & 3 & 64 & 7,0 \\
Psicologia & 9 & 14 & 2 & 9 & 6 & 3 & 2 & 6 & 3 & 6 & 60 & 6,5 \\
Med. Veterinária & - & 1 & 2 & 6 & 1 & 6 & 3 & 10 & 11 & 16 & 56 & 6,1 \\
Pedagogia & 7 & 6 & 5 & 7 & 5 & 3 & 2 & 5 & 2 & 5 & 47 & 5,1 \\
Ciências Sociais & 7 & 1 & 8 & 3 & 1 & - & 1 & 4 & 5 & 5 & 35 & 3,8 \\
Nutrição & 2 & 1 & 2 & - & 1 & - & 3 & 8 & 5 & 1 & 23 & 2,5 \\
Direito & 3 & 4 & 6 & 2 & - & 1 & - & 1 & 3 & 3 & 23 & 2,5 \\
Ciências Biológicas & - & - & 2 & - & 2 & 1 & 1 & 4 & 3 & 1 & 14 & 1,5 \\
\hline Total & 1 & 5 & - & - & - & - & - & 3 & 2 & - & 11 & 1,2 \\
\hline
\end{tabular}

\section{Gráfico 3}

Média da Idade dos Alunos do Curso de Especialização em Saúde Pública da

Universidade de São Paulo, no Periodo 1985-1994

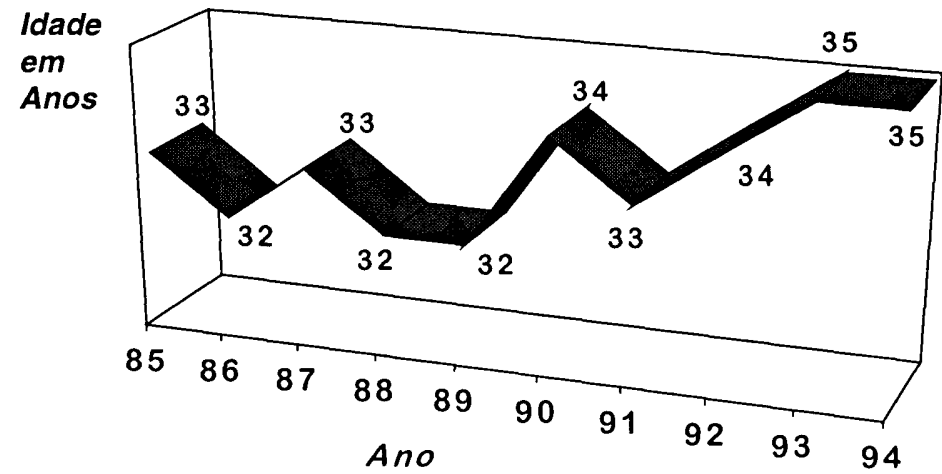

Média $=33 \quad$ Moda $=31 \quad$ Mediana $=32$ 
concluídos os estudos de graduação. A menor idade registrada foi de 22 anos e a maior 57. Quanto à distribuição dos alunos segundo sexo/gênero, $78 \%$ corresponderam ao sexo feminino e apenas 22\%, ao masculino (Gráfico 2). Esses dados podem significar maior sensibilidade das mulheres para os problemas relacionados à saúde pública, mas podem indicar, também - e isso parece o mais provável - que, dados os baixos padrões de remuneração dos profissionais que se dedicam à saúde pública, para esse segmento do mercado de trabalho acabem sendo encaminhadas as mulheres, as quais vêm auferindo menor remuneração no conjunto do mercado de trabalho, conforme o Mapa do Mercado de Trabalho no Brasil (Instituto Brasileiro de Geografia e Estatística - IBGE, 1994).

\section{Considerações finais}

A análise realizada permite concluir que, no período 1985-1994, ocorreram significativas alterações nas características dos alunos do Curso de Especialização em Saúde Pública da Faculdade de Saúde Pública da Universidade de São Paulo, destacando-se o progressivo afastamento da categoria profissional de médicos e o crescente interesse dos assistentes sociais e psicólogos. Verifica-se também que a USP, outrora formadora de especialistas em Saúde Pública vindos das mais diferentes macrorregiões brasileiras e mesmo do exterior, não tem conseguido manter tal abrangência uma vez que a clientela do CESP vem se restringindo, gradativamente, a alunos oriundos do município de São Paulo. Tal característica parece resultar, também, do fato de ter havido, nos anos 70 e 80 , significativa expansão no número de cursos de especialização em Saúde Pública, os quais passaram a se desenvolver em praticamente todas as macrorregiões do Brasil.

Articuladas ao processo histórico de formação de especialistas em Saúde Pública pela
FSP-USP, as informações apresentadas e a análise realizada permitem afirmar que o período que se inicia após a II Guerra Mundial, e vai até o final dos anos 70 , é marcado pelo surgimento de cursos destinados à especialização de certas categorias profissionais. Ocorre uma espécie de "expansão" da experiência que se iniciou em 1927 com a categoria dos médicos: seria a vez dos engenheiros (1949), veterinários (1954), dentistas (1958), enfermeiros (1959) e outros. Em 1982, registra-se a operação de unificação desses cursos, com o objetivo de preparar profissionais de saúde, independentemente de sua formação (graduação), para as funções de sanitarista demandadas pelo Sistema de Saúde brasileiro. As mudanças em curso no sistema, intensificadas com o processo de descentralização e reorganização que ganharam expressão formal com a criação do Sistema Único de Saúde (SUS), em 1988, influiriam decisivamente nos tipos de especialistas em saúde pública requeridos nesse novo contexto histórico. $O$ antigo sanitarista, em cuja formação têm insistido boa parte dos cursos de especialização em Saúde Pública, inclusive o CESP, vem encontrando crescentes dificuldades de inserção nas organizações sanitárias surgidas com a municipalização do sistema. Em conseqüência, o CESP, e cursos similares, vêm perdendo a sintonia com as novas realidades.

Cabe salientar, a propósito, além dos limites deste estudo em particular, os da abordagem utilizada para a compreensão e análise das questões relativas a cursos de especialização em Saúde Pública, as quais requerem enfoques que permitam ir além dos aspectos meramente descritivos, aqui enfatizados. Considera-se necessário, também, dar tratamento adequado aos problemas relacionados com os aspectos pedagógicos e, principalmente, aos professores envolvidos nesses cursos; aspectos estes comumente ausentes nas análises sobre o ensino em Saúde Pública. 
Assim, no final do século $\mathrm{XX}$, um novo cenário se configura, exigindo da FSP-USP iniciativas inovadoras no ensino em Saúde Pública. Neste cenário, outras exigências são postas pela sociedade aos que atuam nesse campo. Ainda que as múltiplas dimensões das iniciativas requeridas escapem aos propósitos deste trabalho, é pertinente destacar a necessidade de se repensar temas relacionados, entre outros, aos processos de ensino-

\section{Referências bibliográficas}

CANDEIAS, N.M.F. (1984) - Memória Histórica da Faculdade de Saúde Pública da Universidade de São Paulo: 1918-1945. Revista de Saúde Pública 18:2-60.

INSTITUTO BRASILEIRO DE GEOGRAFIA E ESTATÍSTICA (1994) - Mapa do Mercado de Trabalbo no Brasil. Rio de Janeiro: IBGE.

KISIL, M. (1994) - Educaçâo em Administraçâo de Saúde na América Latina: A Busca de um Paradigma. São Paulo: FSP-USP.

KRAUSZ, R.R. (1977) - Algumas Reflexões sobre a Formação de Recursos Humanos para a Saúde Pública. Revista de Saúde Pública 11:151-5.

LABRA, M. E. (1985) - O Movimento Sanitarista nos Anos 20: Da Conexão Sanitária Internacional à Especialização em Saúde Pública no Brasil. Dissertação de Mestrado, Escola Brasileira de Administração Pública da Fundação Getúlio Vargas. Rio de Janeiro, mimeo. aprendizagem; ao alcance ideológico dos conteúdos; aos diversos significados do trabalho do professor/a, educador/a, instrutor/ a; às políticas, às práticas e à organização escolar; às especificidades da educação e ao ensino de adultos; aos impactos das novas tecnologias no processo educacional; e às interfaces das escolas com outros espaços sociais e com os veículos de difusão do conhecimento.

MASCARENHAS, R.S. (1951) - Paula Souza, o Sanitarista Social. Anais de Enfermagem 4(3): 231-40.

TANAKA, A.C.A. (1996) - Perfil da Demanda dos Cursos de Pós-Graduação em Saúde Coletiva no Brasil. São Paulo, mimeo.

UNIVERSIDADE DE SÃO PAULO (1994) - Faculdade de Saúde Pública. Catálogo de Cursos de PósGraduaçâo. São Paulo: USP.

VASCONCELLOS, M.P.C. (1995) - Memórias da Saúde Pública: a Fotografia como Testemunba. São Paulo: Editora Hucitec/ABRASCO.

VASCONCELLOS, M.P.C. \& NARVAI, P.C. (1994) Algumas Características dos Alunos do Curso de Especialização em Saúde Pública da USP no período de 1985-94. In: Universidade de São Paulo. Faculdade de Saúde Pública - Reflexões sobre Revisão Institucional Curricular. São Paulo: FSP-CPG-CE/USP. 